\title{
ВмJ Global Health Practical issues in the measurement of child survival in health systems trials: experience developing a digital community-based mortality surveillance programme in rural Nepal
}

Alex Harsha Bangura, ${ }^{1,2}$ Al Ozonoff, ${ }^{2,3}$ David Citrin, ${ }^{1,4,5,6}$ Poshan Thapa, ${ }^{1}$
Isha Nirola, ${ }^{1}$ Sheela Maru, ${ }^{1,7,8,9}$ Ryan Schwarz, ${ }^{1,10,11,12}$ Anant Raut, ${ }^{1}$
Bishal Belbase, ${ }^{1}$ Scott Halliday, ${ }^{1,6}$ Mukesh Adhikari, ${ }^{13}$ Duncan Maru ${ }^{1,10,11,14}$

To cite: Harsha Bangura A, Ozonoff A, Citrin D, et al. Practical issues in the measurement of child survival in health systems trials: experience developing a digital community-based mortality surveillance programme in rural Nepal. BMJ Global Health 2016;1 e000050. doi:10.1136/ bmjgh-2016-000050

Received 8 March 2016 Revised 24 October 2016 Accepted 25 October 2016

CrossMark

For numbered affiliations see end of article.

Correspondence to Dr Duncan Maru; duncan@possiblehealth.org

\section{ABSTRACT}

Child mortality measurement is essential to the impact evaluation of maternal and child healthcare systems interventions. In the absence of vital statistics systems, however, assessment methodologies for locally relevant interventions are severely challenged. Methods for assessing the under-5 mortality rate for crosscountry comparisons, often used in determining progress towards development targets, pose challenges to implementers and researchers trying to assess the population impact of targeted interventions at more local levels. Here, we discuss the programmatic approach we have taken to mortality measurement in the context of delivering healthcare via a public-private partnership in rural Nepal. Both government officials and the delivery organisation, Possible, felt it was important to understand child mortality at a fine-grain spatial and temporal level. We discuss both the shortterm and the long-term approach. In the short term, the team chose to use the under- 2 mortality rate as a metric for mortality measurement for the following reasons: (1) as overall childhood mortality declines, like it has in rural Nepal, deaths concentrate among children under the age of 2; (2) 2-year cohorts are shorter and thus may show an impact more readily in the short term of intervention trials; and (3) 2-year cohorts are smaller, making prospective census cohorts more feasible in small populations. In the long term, Possible developed a digital continuous surveillance system to capture deaths as they occur, at which point under-5 mortality assessment would be desirable, largely owing to its role as a global standard.

\section{INTRODUCTION}

Improving child survival is a central function of healthcare systems globally. There remains a substantial implementation gap in translating evidence-based interventions into healthcare systems improvements. Trials that test healthcare systems innovations are central to

\section{Key questions}

What is already known about this topic?

- Assessing child mortality is essential to understanding healthcare systems performance.

- Many low-income and middle-income countries lack robust vital registration systems and censuses, which hinders the reporting of accurate mortality rates.

- Child mortality estimates among smaller catchment area populations lack the reliability and precision to inform local or subnational public health programming.

- Implementers in resource-limited settings have a limited toolbox for mortality assessment in rea time and at local levels.

What are the new findings?

- We describe an approach to a digital system for measuring mortality using community health workers. This approach, as is typical for grassroots implementers in resource-limited settings, necessarily proceeds in steps.

- In the initial phase, the team chose the under-2 mortality rate in lieu of the under-5 mortality rate given the baseline lack of strong vital registration systems and censuses, and the greater feasibility and reliability of this metric in our setting.

- Calculating the under-2 mortality rate requires only 2-year birth histories, is subject to less recall bias and can more accurately estimate the distribution of mortality in populations where child mortality is concentrated in the first 2 years of life.

- In the longer term, the team is developing a community health worker-based continuous surveillance system built into the routine course of clinical care for reproductive age women. Active case search allows care to begin for pregnant women soon after conception through the first 2 years of a child's life, or the 'Golden 1000 days'. 


\section{Key questions}

\section{Recommendations for policy}

- In the initial phases of deploying digital community-based methods of mortality assessment, the under- 2 mortality rate may be an appropriate metric in some settings.

- This initial phase can then form the basis of a continuous surveillance system to track child mortality among small populations at the subnational level. Such information can inform public health programming and healthcare delivery.

- Developing geospatially and temporally sensitive population health surveillance systems can approximate 'real-time' vital registration that allows for mortality assessment of any age group with greater precision and accuracy.

meeting this gap. The routine measurement of key population health outcomes, such as child mortality, presents a major challenge in implementation research on complex healthcare systems interventions in resourcelimited settings.

It is essential that countries and individual programmes develop methods to continuously assess deaths, preferably within formal vital statistics systems. Continuous surveillance systems relying on local community health workers (CHWs) are feasible, as has been shown in one severely resource-constrained environment in Mali. ${ }^{1}$ In the modern healthcare era, these systems should be digitised and integrated within routine longitudinal healthcare delivery. Yet it is unclear how to best create digital surveillance systems from current paper registry and intermittent survey approach.

Here, we explore, from the grassroots view of public sector healthcare implementers in rural Nepal, the path to develop more robust, locally responsive and meaningful measures of child mortality. We describe the direction that the Government of Nepal and the non-profit organisation, Possible, has taken via a public-private partnership (PPP) to achieve the objective of feasible and rigorous outcomes assessments of child survival initiatives.

Possible is a non-profit organisation that works with the Ministry of Health of Nepal via a PPP to run an integrated hospital and CHW network. In this approach, the government provides facilities, staff, supplies and co-financing, and Possible assumes management authority and is held accountable for direct healthcare delivery. Through this PPP, the team is committed to developing an implementation research programme that can be used for local and national healthcare systems learning and improvement. Child mortality is a key indicator for their implementation research programme.

The PPP team has confronted the challenge of child mortality estimation in Achham, a rural district of 260000 people in the Far-Western Development Region of Nepal. Lying 30 hours from the capital city, with some of the lowest development indicators in the country, and emerging from a civil war in which it was one of the key conflict centres, Achham poses great challenges to healthcare systems strengthening..$^{2}$ In Achham, like in much of the developing world, one of the major barriers to measuring accurate and spatiotemporally precise population-based metrics is infrequent census updates (usually every 10 years) and the absence of effective vital registration systems. In Achham, $\sim 35 \%$ of children under-5 have birth certificates, compared with a national average of $60 \% .^{34}$

Given these challenges of working in resource-limited rural Nepal, the PPP team realised that it was important to take a stepwise approach to assess child mortality. In the first phase, the team felt that the under-2 mortality rate (U2MR) could be more feasibly used as the basis for similar healthcare systems trials with small population sizes. We describe the PPP team's experiences in assessing this measure in rural Nepal through a census survey and our work towards continuous surveillance methods that can approximate a vital registration system.

\section{Child mortality estimation: practices and pitfalls}

The under-5 mortality rate (U5MR) has been broadly applied as a metric to assess progress in reducing child mortality across countries and over time for many years. It was the basis of Millennium Development Goal (MDG) 4 and the recently outlined Sustainable Development Goal (SDG) 3, which aims to reduce the U5MR to $<25$ deaths per 1000 live births. While useful for cross-country comparisons, the U5MR poses problems in collection and analysis for intervention trials, particularly in small populations and for assessment over shorter (eg, yearly) time periods.

In the absence of vital registration systems, estimates of child mortality are created by surveying mothers about their children, both living and deceased, with either: (1) the direct method using full birth histories, which include birth and death dates for each child, or (2) the indirect method using summary birth histories (all children ever born and ever died) and some proxy measure of exposure (ie, mother's age or time since first birth) to impute the ages of those children. ${ }^{5}$ Most sources of national mortality estimates use the full birth history method as it most closely replicates a vital registration system, if one assumes that coverage and recall are not biased. Collection of full birth histories is timeconsuming, however, and the results are susceptible to so-called 'age heaping' around 12 months, which biases estimates of infant mortality upwards. ${ }^{7}$ Similarly, birth transference to ages older than 5 to avoid completion of the under-5 data collection contributes to concerns over data quality for the full birth history method. ${ }^{8}$

The indirect method relies on life tables to impute ages and estimate overall mortality risk before age 5 , and then to derive infant mortality. ${ }^{5}$ This is more vulnerable to inaccurate fertility and mortality assumptions. ${ }^{7}$ Indirect methods may exclude data from the youngest mothers (ages 15-24 years) because children born to these mothers are high-risk first births and are most likely from lower socioeconomic groups. ${ }^{7}$ Since these 
mothers have the highest proportion of young children, their exclusion means both that recent mortality estimates cannot be reliably calculated and, perhaps equally as important, the estimate no longer reflects the true mortality risk of children born to those women. ${ }^{9}$ When the goal is to measure the impact of specific interventions on reduction of mortality risk, introducing these known biases is problematic.

These challenges are less significant on a national or global scale, as the methods have been developed explicitly for the purpose of comparing general trends across countries and multiyear time frames rather than specific point estimates. When considering the role of mortality estimation in subnational interventions, however, particularly those in need of holistic measures of healthcare systems improvement, child mortality estimation becomes especially problematic. The U5MR derived via national Demographic Health Surveys does not typically offer the precision necessary to find statistically significant differences across time or subnational populations. ${ }^{10}{ }^{11}$ Estimation of mortality for recent periods (ie, within the 5 years preceding the survey) requires either strong assumptions about underlying fertility and mortality trends for indirect methods or large sample sizes for indirect and direct methods that make it untenable in small populations of 15000 households or less when the U5MR dips below 100. ${ }^{8} 1012$ Furthermore, U5MR estimates do not provide information regarding age distribution of deaths relevant for targeted public health programming. However, estimating mortality for different age groups is possible from full birth history data.

\section{Context of child mortality in Nepal}

Since the MDG era has passed, the global focus has shifted from addressing under-5 mortality to infant and neonatal mortality. ${ }^{13}$ The infant mortality rate (IMR) and neonatal mortality rate (NMR) are defined as the number of deaths per 1000 live births occurring before year 1 and before 28 days, respectively. In 2011, Nepal reached its U5MR target of 54, but neonatal and infant mortality improvements continue to lag behind overall under-5 mortality success. ${ }^{14}$ This pattern largely reflects a stagnant NMR over the same period, suggestive of the many challenges in addressing perinatal and infant mortality due to complications such as birth asphyxia, low birth weight and malnutrition.

The pattern of under-5 mortality decline in Nepal is not unique. Historically, transitions in childhood mortality have imparted significant changes in the distribution of deaths. Since the overall U5MR has decreased, the proportion of deaths occurring in the perinatal and neonatal periods has increased. For example, when the U5MR estimate is $<60, \sim 75 \%$ of deaths are concentrated in the infant period, with an NMR/U5MR ratio of $>0.5 .^{7}$ Deaths caused by diarrhoea and lower respiratory tract illness decrease almost linearly with a decreasing U5MR, while deaths from vaccine-preventable illness, perinatal and other causes remain stable at $25-35 \%$ of total
Under-5 deaths. ${ }^{15}$ Thus, the proportion of infant ( $<1$ year) versus child ( $1-5$ years) mortality reflects both programmatically and epidemiologically relevant trends regarding underlying causes of death and the current state of the healthcare system. The U5MR itself does not provide a breakdown of mortality by age, however, and could reflect many combinations of infant and child mortality. Focusing solely on infant mortality also has drawbacks as there is commonly under-reporting of infant deaths, age heaping around 12 months and the cohort represents yet a smaller population for which sampling error is more problematic. ${ }^{7}$

Considering that the prioritisation of health programming in Nepal includes newborn health and the 'Golden 1000 days' (conception to age 2), ${ }^{16}$ and the fact that over $80 \%$ of under-5 deaths occur under age $2,{ }^{3}$ the PPP team determined that the U2MR was more appropriate than the U5MR. The PPP team decided that the U2MR would provide a more robust and relevant estimate for implementation research trials in rural Nepal. Two-year cohorts emphasise neonatal mortality risk but also include some more exposure time during which infectious diseases are a major cause of death. Like the U5MR, the larger cohort may attenuate problems with age heaping and sampling error associated with the IMR. With a relatively short estimation window, since children age out of the cohort faster, the U2MR may also be valuable for iterative improvement in intervention design. Whether for under-2 or under-5 mortality, in small populations, sample surveys are often underpowered and census methods more appropriate. For example, a traditional survey aiming to assess a $20 \%$ change in under-5 mortality requires a sample of nearly 7000 women which, in the context of the hospital's catchment area population, is essentially the entire eligible population. $^{12} 17$

Given the significant resource constraints in the first iteration, the PPP team chose a truncated birth history method, collecting the birth and death dates of all children born in the prior 2 years during the first phase of data collection. In May 2015, the PPP team completed this first census phase to establish baselines for the U2MR, IMR and NMR, as well as institutional birth rates, antenatal care coverage and postpartum contraceptive prevalence rates. They adapted the survey instrument from the Demographic and Health Surveys and Multiple Indicator Cluster Survey, ${ }^{18}$ for an Android-based mobile phone running an Open Data Kit application. ${ }^{19}$ The PPP team translated the tools into Nepali and stored the data on SurveyCTO's secure, cloud-based servers. ${ }^{20} \mathrm{CHWs}$, known locally in Nepal as Female Community Health Volunteers, registered and mapped over 7500 households and collected targeted birth histories from all 1223 women reporting a birth in the previous 2 years. This approach achieved $\sim 70 \%$ coverage of the eligible population, as $15 \%$ of households and $20 \%$ of identified women were unavailable due to migration or work responsibilities. 
In the initial data set of 1237 births, the number of reported under-2 deaths was surprisingly low. The estimated U2MR was 36.9 per 1000 live births, with all reported deaths before or at 1 year of age. That no deaths occurred after age 1 suggested either: (1) birth transference, in which women or interviewers deliberately report a child as older than the age cut-off in order to avoid answering questions, which may have biased the mortality estimate downwards, or (2) mortality is lower than previously thought. There is a dearth of data on the validity of truncated birth history methods, however, and it is unclear whether shortening the window of eligible births would improve recall or birth transference bias. Given the intent to transition to continuous surveillance, the PPP team did not formally validate these methods but may have the opportunity to compare the initial estimates with repeated measures in the coming years.

\section{Moving towards continuous surveillance}

Since one key aim of the PPP is to develop an implementation research programme that is responsive and sustainable for ongoing healthcare systems innovation and care delivery, mortality estimation methods should ultimately be designed for continuous surveillance. With continuous surveillance of the entire population, any combination of mortality indicators could theoretically be calculated. In the initial phases of mortality assessment for healthcare systems design, the PPP chose the U2MR as the highest value indicator both for applicability and practicability. Based on the Government of Nepal's projections for the hospital's catchment area population, $\sim 10 \%$ of women of childbearing age deliver a child every year. ${ }^{3}$ Given the recent data that Nepali women space births by a median of 2-3 years and have an average of three children, an estimated $16-18 \%$ of women have children under age 2, 35-40\% have children under age 5 and $90 \%$ have ever had children. ${ }^{21}$ Continuous surveillance of all women with children under age 5 would thus more than double the women followed, overburdening healthcare workers who have primary patient care responsibilities with most likely a small impact on overall child survival.

Outside of Nepal, real-time monitoring of vital events has been proposed as an alternative method of child mortality assessment. Several demographic surveillance sites globally have demonstrated success through intensive routine measurement. ${ }^{22}$ Over the past 50 years, the INDEPTH network has developed health and demographic surveillance systems in 19 countries around the world, often leveraging CHWs for birth and death registration, as well as continuous surveillance of maternal and child health status and chronic disease morbidity. ${ }^{23}$ Recent studies have demonstrated the method's success at generating data sets robust to yearly mortality estimates at the village level that can be used for spatiotemporal analysis of disparity in trends. ${ }^{24-26}$
In non-health and demographic surveillance systems sites, vital events reporting has often been a responsibility of national CHW programmes, but studies validating these data sources are few. The Real-Time Monitoring of Under-Five Mortality Project recently reported mixed success with their five-country trial of strengthened CHW vital events reporting, suggesting that successful programmes were more resource-intensive than what would likely be sustainable in similar settings. ${ }^{1}$ 27-30

To improve sustainability and scalability, the PPP team thus endeavoured to develop continuous vital events surveillance within an electronic medical record. This is implemented through a CHW network and a mobile application for care coordination (CommCare, developed by Dimagi) ${ }^{31} 32$ integrated with an existing hospitalbased electronic medical record (OpenMRS-based Bahmni, developed by Thoughtworks). The electronic medical record product integrates and strengthens the core surveillance and care functions of the CHW by embedding active pregnancy case-finding and mortality surveillance into community-based care, which already aims to touch every eligible household. ${ }^{25}$

\section{Integration of continuous surveillance and routine care through a mobile electronic medical records system}

Before beginning continuous surveillance, the PPP team attempted to visit previously missing households and women to finalise the census roll. It quickly became clear, however, that inadequate identification of households in the first phase would make this largely impossible. Working with other local government officials, the PPP team re-enumerated all households (including truncated birth histories for eligible women) with durable markers (small tin plaques with unique household IDs attached to homes). Together with the organisation SimPrints, ${ }^{33}$ the team improved on the case management application with biometrics integration for fingerprinting identification of mothers and children. This was successfully pilot-tested using the biometrics tool with community members, with promising preliminary results showing that the use of two-finger identification in the cohort correctly returned a person as ranked first in search results about $97 \%$ of the time. This accuracy is also demonstrated before optimisation using the household Global Positioning System coordinates, or searching with other fingers. The mobile surveillance tool was fully deployed in May 2016.

In the revised system, CHWs visit every household on a quarterly basis to screen eligible women (married, aged 15-49) for early pregnancy, based on an algorithm for identifying unexplained amenorrhoea of $>6$ weeks duration. If women screen positive, they are offered home pregnancy testing and ultimately enrolled in home antenatal visits and group antenatal care offered at local clinics. CHWs document births and deaths during routine visits where they provide pregnant women and children under age 2 with preventive care and referrals for group antenatal and paediatric care or 


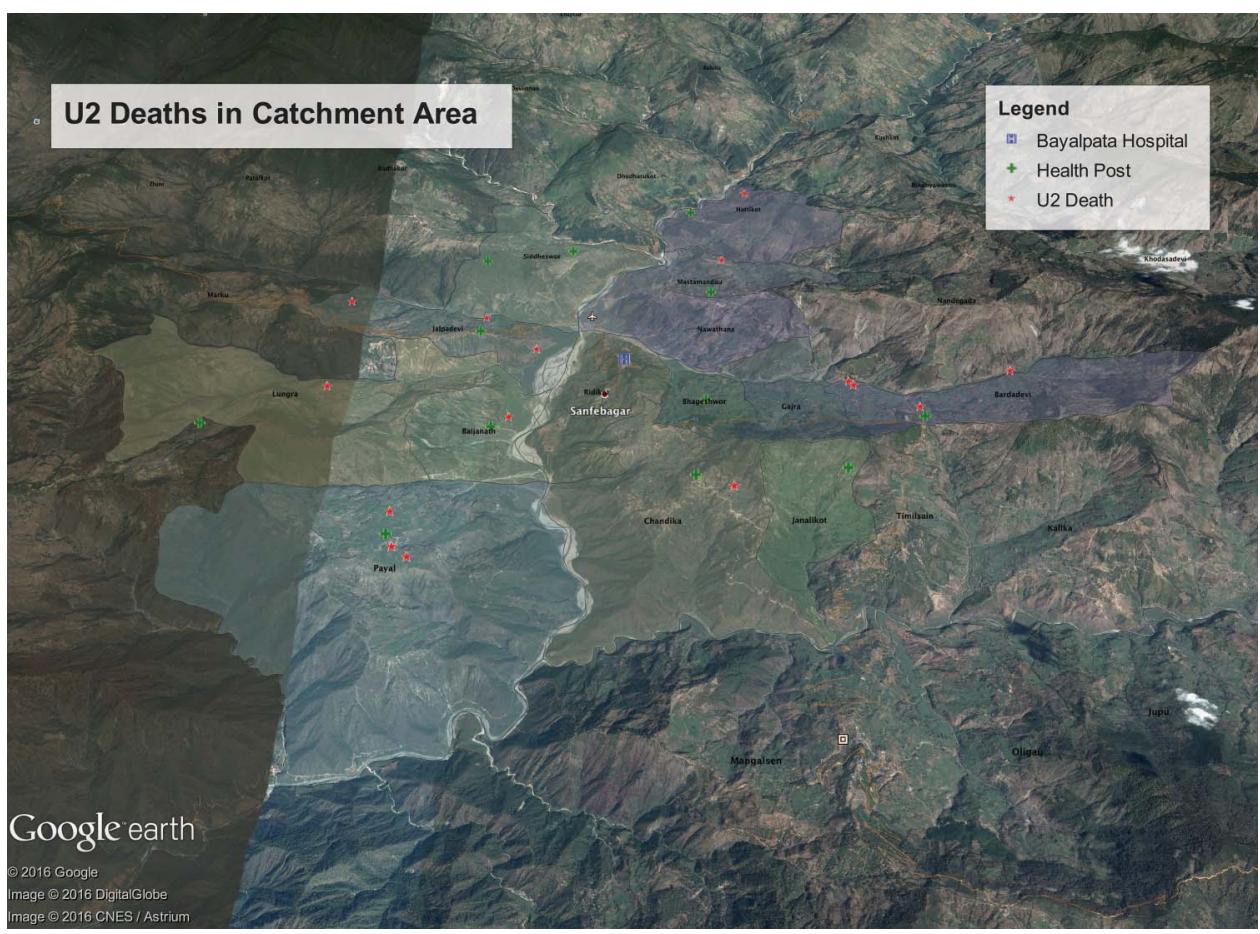

Figure 1 Under-2 (U2) deaths in the catchment area.

urgent facility-based services. These data are documented with mobile forms integrated into the electronic medical record, as described above. Figure 1 is a geotopographical map that visualises under-2 deaths collected through the continuous surveillance system. Through this routine outreach, the goal is to create a relatively real-time vital registration system that enables mortality assessment with spatiotemporal precision and accuracy. Over time, a verbal autopsy programme will be implemented to assess the cause of death through standardised family interviews, ${ }^{34-36}$ further deepening an understanding of healthcare system gaps.

\section{CONCLUSION}

Entering the post-MDG era and moving into the SDGs, the focus of mortality measurements must shift towards the development of robust impact assessments that are relevant to changing mortality patterns and feasible for community-level programme implementation. Given the relatively rare nature of childhood mortality and the absence of effective vital registration systems in much of the developing world, the precision of child mortality estimation is greatly affected by sample size and estimates cannot easily be disaggregated to district or subdistrict levels. As overall childhood mortality declines, deaths concentrate in the neonatal and infant periods, and there is substantial geographic clustering and inequities that only fine-grained data can capture.

The PPP team's initial experience with a census-based truncated birth history highlights the challenges of retrospective data collection when counting every death. Using an integrated, biometrics-enabled mobile application, the continuous surveillance system follows children through age 2 , thus feasibly measuring under-2 mortality to inform public health programming and implementation research studies on child survival. Eventually, children under-5, under-10 or even the entire population (eg, for chronic diseases primarily affecting adults) can be continuously followed up and monitored in this system. Key questions will be around affordability, training, privacy and government and community buy-in. We aim to explore the validity, costs and sustainability of such a system in the coming years. In the mean time, we encourage others to explore how measurement of vital events might be integrated into routine community healthcare delivery in other settings and the effect of similar or unique technologies on data quality and efficiency.

\section{Author affiliations}

${ }^{1}$ Possible, Bayalpata Hospital, Sanfebagar-10, Achham, Nepal

${ }^{2}$ Harvard Medical School, Boston, MA, USA

${ }^{3}$ Boston Children's Hospital, Center for Patient Safety and Quality Research, Boston, MA, USA

${ }^{4}$ University of Washington, Department of Anthropology, Seattle, WA, USA ${ }^{5}$ University of Washington, Department of Global Health, Seattle, WA, USA

${ }^{6}$ University of Washington, Henry M. Jackson School of International Studies, Seattle, WA, USA

${ }^{7}$ Boston Medical Center, Department of Obstetrics and Gynecology, Boston, MA, USA

${ }^{8}$ Boston University School of Medicine, Department of Obstetrics and Gynecology, Boston, MA, USA

${ }^{9}$ Brigham and Women's Hospital, Department Medicine, Division of Women's Health, Boston, MA, USA

${ }^{10}$ Brigham and Women's Hospital, Department of Medicine, Division of Global Health Equity, Boston, MA, USA

${ }^{11}$ Harvard Medical School, Department of Medicine, Boston, MA, USA 
${ }^{12}$ Massachusetts General Hospital, Department of Medicine, Division of General Internal Medicine, Boston, MA, USA

${ }^{13}$ Ministry of Health, Department of Health Services, District Health Office, Mangalsen, Achham, Nepal

${ }^{14}$ Boston Children's Hospital, Department of Medicine, Division of General Pediatrics, Boston, MA, USA

\section{Handling editor Seye Abimbola.}

Acknowledgements The authors wish to express their appreciation to the Nepal Ministry of Health for their continued efforts to improve access to healthcare in rural Nepal and for their engagement on critically examining child mortality assessment methods. The authors wish to thank Sachit Pandey and Andrea Morin for assistance with preparing figure 1. They also wish to express their deepest appreciation to their partners at the Achham District Health Office and Public Health Office as well as all of the allied healthcare workers serving Achham District including the Female Community Health Volunteers, (Sub) Health Post and Primary Care Center staff members, and Achham District Health Office staff members. By continuing to provide excellent and compassionate healthcare to the citizens of Achham, you are the catalysts for reducing child mortality in the communities.

Contributors AHB, AO and DM conceived of conceptual basis for U2MR as child mortality assessment metric. All authors contributed to design, refactoring and critical evaluation of continuous surveillance census system. AHB, DC, PT, IN, SM, RS, AR, BB, SH and DM implemented continuous surveillance census system. AHB, AO, DC, SM, PT and DM contributed to the writing of the manuscript. ICMJE criteria for authorship was read and met by all authors. All authors agree with manuscript results and conclusions.

Funding Research reported in this publication was supported by the Office of the Director, National Institutes of Health of under award number DP50D019894. The Eunice Kennedy Shriver National Institute of Child Health \& Human Development (NICHD) and the National Institute of Dental \& Craniofacial Research (NIDCR) provided support for this award. Research reported in this publication was also supported by the Charles $\mathrm{H}$ Hood Foundation. The Center for Primary Care at Harvard Medical School provided funding support for Dr Alex Harsha Bangura. The funders had no role in the program design, program implementation, decision to publish, or preparation of the manuscript.

Disclaimer The content is solely the responsibility of the authors and does not necessarily represent the official views of the National Institutes of Health.

Competing interests $A H B, S M, R S$, and DM work in partnership with and $\mathrm{DC}, \mathrm{PT}, \mathrm{IN}, \mathrm{AR}, \mathrm{BB}$, and $\mathrm{SH}$ are employed by a nonprofit healthcare company (Possible) that delivers free healthcare in rural Nepal using funds from the Government of Nepal and other public, philanthropic, and private foundation sources. AHB is a medical student at, and AO, RS, and DM are faculty members at a private university (Harvard Medical School). AO and DM are employed at academic medical centers (Boston Children's Hospital) that receive public sector research funding, as well as revenue through private sector fee-for-service medical transactions and private foundation grants. DC is a faculty member at and both DC and SH are employed part-time by a public university (University of Washington). SM is employed at an academic medical center (Boston Medical Center) that receive public sector research funding, as well as revenue through private sector fee-for-service medical transactions and private foundation grants. SM is a faculty member at a private university (Boston University). SM is a research fellow at and both RS and DM are employed by an academic medical center (Brigham and Women's Hospital) that receives public sector research funding, as well as revenue through private sector fee-for-service medical transactions and private foundation grants. RS is also employed at an academic medical center (Massachusetts General Hospital) that receives public sector research funding, as well as revenue through private sector fee-for-service medical transactions and private foundation grants. MA is employed by the Government of Nepal. DM is a non-voting member on Possible's board of directors, but receives no compensation. All authors have read and understood BMJ Global Health's policy on declaration of interests, and declare that we have no competing financial interests. The authors do, however, believe strongly that healthcare is a public good, not a private commodity.
Provenance and peer review Not commissioned; externally peer reviewed.

Data sharing statement De-identified quantitative data are available at the Healthcare System Design Group's (Possible's Implementation Research Team) website (http://hsdg.partners.org/data/). Data may also be requested by emailing: research@possiblehealth.org.

Open Access This is an Open Access article distributed in accordance with the Creative Commons Attribution Non Commercial (CC BY-NC 4.0) license, which permits others to distribute, remix, adapt, build upon this work noncommercially, and license their derivative works on different terms, provided the original work is properly cited and the use is non-commercial. See: http:// creativecommons.org/licenses/by-nc/4.0/

\section{REFERENCES}

1. Munos MK, Koffi AK, Sangho $H$, et al. Strengthening community networks for vital event reporting: community-based reporting of vital events in rural Mali. PLOS ONE 2015;10:e0132164.

2. Government of Nepal, National Planning Commission, United Nations Development Programme. Nepal Human Development Report 2014: beyond geography, unlocking human potential. Kathmandu, Nepal: 2014

3. Ministry of Health and Population, New ERA, ICF International Inc. Nepal Demographic and Health Survey 2011. Kathmandu, Nepal: Ministry of Health and Population, New ERA, ICF International Inc, 2012.

4. United Nations Children's Fund. Every Child's Birth Right: inequities and trends in birth registration. New York: UNICEF, 2013.

5. Hill K, You D, Inoue M, et al. Technical Advisory Group of the United Nations Inter-agency Group for Child Mortality E. Child mortality estimation: accelerated progress in reducing global child mortality, 1990-2010. PLoS Med 2012;9:e1001303.

6. UNICEF, World Health Organization. The World Bank, United Nations Levels and Trends in Child Mortality: estimates developed by the UN Inter-agency Group for Child Mortality Estimation. New York: UNICEF, 2012. http://www.who.int/maternal_child_ adolescent/documents/levels_trends_child_mortality_2012.pdf.

7. Guillot M, Gerland P, Pelletier F, et al. Child mortality estimation: a global overview of infant and child mortality age patterns in light of new empirical data. PLoS Med 2012;9:e1001299.

8. Silva R. Child mortality estimation: consistency of under-five mortality rate estimates using full birth histories and summary birth histories. PLoS Med 2012;9:e1001296.

9. Rajaratnam JK, Tran LN, Lopez AD, et al. Measuring under-five mortality: validation of new low-cost methods. PLoS Med 2010;7: e1000253.

10. Pedersen J, Liu J. Child mortality estimation: appropriate time periods for child mortality estimates from full birth histories. PLoS Med 2012;9:e1001289.

11. Alkema L, New JR. Progress toward global reduction in under-five mortality: a bootstrap analysis of uncertainty in millennium development goal 4 estimates. PLoS Med 2012;9:e1001355.

12. Korenromp EL, Arnold F, Williams BG, et al. Monitoring trends in under-5 mortality rates through national birth history surveys. Int $J$ Epidemiol 2004;33:1293-301.

13. Ministry of Health and Population, Department of Health Services. Annual report, Department of Health Services 2070/20171 (20132014). Teku, Kathmandu: Ministry of Health and Population DoHS, 2014.

14. Government of Nepal, National Planning Commission, United Nations Country Team Nepal. Nepal Millennium Development Goals, Progress Report 2013. Kathmandu: Government of Nepal, National Planning Commission, United Nations Country Team Nepal, 2013.

15. Rao C, Adair T, Kinfu Y. Using historical vital statistics to predict the distribution of under-five mortality by cause. Clin Med Res 2011:9:66.

16. Government of Nepal, National Planning Commission. Multi-sector Nutrition Plan, for accelerating the reduction of maternal and child under-nutrition in Nepal 2013-2017 (2023). Kathmandu: Government of Nepal NPC, 2012.

17. Ministry of Health and Population, Department of Health Services, Health Management Information Section. Estimated Target Population Fiscal Year 2071/72 (2014/2015). Kathmandu, Nepal, 2014. https://data.humdata.org/dataset/hmis-estimated-populationdata-2014-2015

18. Hancioglu A, Arnold F. Measuring coverage in $\mathrm{MNCH}$ : tracking progress in health for women and children using DHS and MICS household surveys. PLoS Med 2013;10:e1001391. 
19. Hartung C, Anokwa Y, Brunette W, et al. Open Data Kit: tools to build information services for developing regions. ICTD, 2010.

20. SurveyCTO. Secondary SurveyCTO. http://www.surveycto.com/ index.html (accessed 8 Sep 2015).

21. Rutstein SO. ICF Macro. Trends in birth spacing. Calverton, MD: Macro I, 2011.

22. Sankoh O, Byass P. The INDEPTH Network: filling vital gaps in global epidemiology. Int J Epidemiol 2012;41:579-88

23. INDEPTH Network. Secondary INDEPTH Network. http://www. indepth-network.org/\#

24. Sankoh OA, Yé Y, Sauerborn R, et al. Clustering of childhood mortality in rural Burkina Faso. Int J Epidemiol 2001;30:485-92.

25. Kant S, Misra P, Gupta S, et al. The Ballabhgarh health and demographic surveillance system (CRHSP-AllMS). Int J Epidemiol 2013;42:758-68.

26. Chin B, Montana L, Basagaña X. Spatial modeling of geographic inequalities in infant and child mortality across Nepal. Health Place 2011:17:929-36.

27. Silva R, Amouzou A, Munos M, et al. Can community health workers report accurately on births and deaths? Results of field assessments in Ethiopia, Malawi and Mali. PLoS ONE 2016;11:e0144662.

28. Amouzou A, Kidanu A, Taddesse N, et al. Using health extension workers for monitoring child mortality in real-time: validation against household survey data in Rural Ethiopia. PLOS ONE 2015;10: e0126909.
29. Joos O, Amouzou A, Silva R, et al. Strengthening community-based vital events reporting for real-time monitoring of under-five mortality: lessons learned from the Balaka and Salima Districts in Malawi. PLoS ONE 2016;11:e0138406.

30. Joos O, Silva R, Amouzou A, et al. Evaluation of a mHealth data quality intervention to improve documentation of pregnancy outcomes by health surveillance assistants in Malawi: a cluster randomized trial. PLOS ONE 2016;11:e0145238.

31. Chatfield A, Javetski G, Lesh N. Dimagi, Inc. CommCare Evidence Base. Cambridge, MA, 2013.

32. Thoughtworks, Inc. Bahmni, Hospital System for Low Resource Settings. Bangalore, India, 2016.

33. SimPrints. Mobile Biometrics for Development. Secondary Mobile Biometrics for Development. http://www.simprints.com (accessed 31 Jan 2016)

34. Engmann C, Jehan I, Ditekemena J, et al. An alternative strategy for perinatal verbal autopsy coding: single versus multiple coders. Trop Med Int Health 2011;16:18-29.

35. Lawn JE, Osrin D, Adler A, et al. Four million neonatal deaths: counting and attribution of cause of death. Paediatr Perinat Epidemiol 2008;22:410-16.

36. Murray CJ, Lopez AD, Black R, et al. Population Health Metrics Research Consortium gold standard verbal autopsy validation study: design, implementation, and development of analysis datasets. Popul Health Metr 2011;9:27. 